\title{
Improved DFT Potential Energy Surfaces via Improved Densities
}

\author{
Min-Cheol Kim, ${ }^{\dagger}$ Hansol Park, ${ }^{\dagger}$ Suyeon Son, ${ }^{\dagger}$ Eunji Sim, ${ }^{* \dagger}$ and Kieron Burke ${ }^{\ddagger}$ \\ ${ }^{\dagger}$ Department of Chemistry and Institute of Nano-Bio Molecular Assemblies, Yonsei University, 50 Yonsei-ro, Seodaemun-gu, Seoul \\ 120-749, Korea \\ ${ }^{\ddagger}$ Department of Chemistry, University of California, 1102 Natural Sciences 2, Irvine, California 92697, United States
}

\section{Supporting Information}

ABSTRACT: Density-corrected DFT is a method that cures several failures of self-consistent semilocal DFT calculations by using a more accurate density instead. A novel procedure employs the Hartree-Fock density to bonds that are more severely stretched than ever before. This substantially increases the range of accurate potential energy surfaces obtainable from semilocal DFT for many heteronuclear molecules. We show that this works for both neutral and charged molecules. We explain why and explore more difficult cases, for example, $\mathrm{CH}^{+}$, where densitycorrected DFT results are even better than sophisticated methods like CCSD. We give a simple criterion for when DC-DFT should be more accurate than self-consistent DFT that can be applied for most cases.

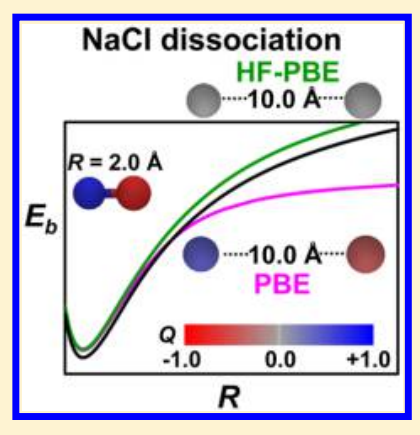

emilocal density functional theory (DFT) has become a $\checkmark$ popular workhorse for electronic structure calculations, despite its many known shortcomings. ${ }^{1}$ A major failure has always been the stretching of bonds into dissociated fragments. Prototypes of this failure are stretched $\mathrm{H}_{2}{ }^{+}$and stretched $\mathrm{H}_{2}$, for which semilocal DFT binding energies generically fail to vanish at large separations. ${ }^{2}$ The identification of selfinteraction error for the former case and static correlation for the latter has been unified in the generic concept of delocalization error of semilocal functionals. ${ }^{3}$

Our focus here is dissociation of heteronuclear diatomic molecular species. In many cases, semilocal DFT dissociates incorrectly into charged fragments, so that the potential energy curve tends to an incorrect value for large $R$, where $R$ is the interatomic distance. ${ }^{4}$ Figure 1 shows the prototypical case, the binding energy curve of $\mathrm{NaCl}$ with standard approximations $\left(\mathrm{PBE}^{5}\right.$ as a generic GGA functional, and B2PLYP, ${ }^{6}$ a double hybrid functional). Beyond about $R=4 \AA$, the PBE curve becomes highly inaccurate, as it tends to an incorrect dissociation limit, $\sim 1 \mathrm{eV}$ below the sum of $\mathrm{Na}$ and $\mathrm{Cl}$ atomic energies in PBE. Even a highly accurate double-hybrid functional such as $\mathrm{B}^{2} \mathrm{PLYP}^{6}$ goes to an incorrect dissociation limit of about $-0.7 \mathrm{eV}$. The work of ref 4 on density-corrected DFT (DC-DFT) showed that by using Hartree-Fock (HF) densities in DC-DFT (which is denoted as HF-DFT) accuracy could be maintained until 5.6 A. At that point the HF energy of neutral fragments dips below that of charged fragments, and the HF-DFT curves abruptly jump to zero. This is shown by the light-blue line for the PBE functional.

In the present work, we correct the procedure for applying HF-DFT to this problem and show that it substantially improves results. This procedure yields the solid blue line of Figure 1, which remains smooth and relatively accurate out to a remarkable 8.2 ̊. We explain why this happens and that it is no accident. We show that a generalization applies to both

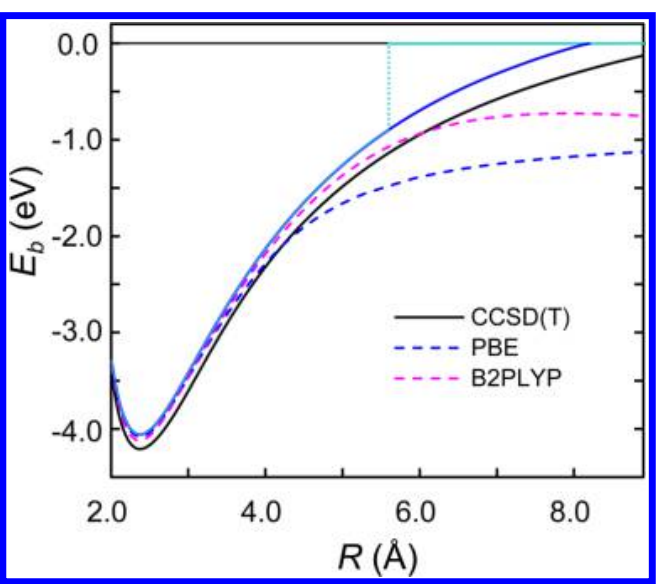

Figure 1. Binding energy curve of $\mathrm{NaCl}$ molecule with various methods. Dashed lines are self-consistent DFT and solid blue line is HF-PBE. The cyan line is HF-PBE with the less useful procedure of ref 4.

molecular anions and cations. We also show some exceptions and how these can still be understood in terms of the basic concepts of DC-DFT. We give a simple criterion for when DCDFT should improve results over self-consistent DFT. Thus, using our simple procedure, the potential energy surface of most heteronuclear diatomics can be greatly improved.

The theory behind DC-DFT is very straightforward. ${ }^{7}$ In a Kohn-Sham DFT (KS-DFT) calculation, ${ }^{8}$ the unknown piece of the energy, the exchange-correlation energy, $E_{\mathrm{XC}}[\rho]$, is approximated, $E_{\mathrm{XC}}^{\mathrm{app}}[\rho]$. The self-consistent solution of the KS

Received: August 7, 2015

Accepted: September 8, 2015 
equations then yields the density that minimizes the total energy, $\rho^{\mathrm{app}}(\mathbf{r})$, and the calculated approximate total energy is $E^{\text {app }}\left[\rho^{\text {app }}\right]$. We decompose the error in the energy by writing

$$
\Delta E=\left(E^{\mathrm{app}}[\rho]-E\right)+\left(E^{\mathrm{app}}\left[\rho^{\mathrm{app}}\right]-E^{\mathrm{app}}[\rho]\right)
$$

The first term is the functional error and is the error made in the energy even if we had used the exact density in our approximate functional. The second is the density-driven error and is the error in the energy due solely to the approximate density. In typical semilocal DFT calculations, the second term is much smaller than the first, and these are called normal. ${ }^{9}$ In unusual and interesting cases, the second-term becomes comparable or even dominates, and these are abnormal. In such cases, evaluation of the semilocal approximation on a more accurate density yields a significant reduction in the energy error. This is DC-DFT. ${ }^{7}$ For many density-driven errors of semilocal DFT calculations, HF densities are sufficient to make the density-driven error negligible.

These effects have been noticed since the start of KS-DFT, ${ }^{10}$ especially in the case of atomic anions, ${ }^{11}$ where the effect of the density-driven error is severe. Some of the earliest DFT calculations in quantum chemistry actually used HF densities ${ }^{12}$ to compare apples-to-apples when testing approximate functionals, but self-consistent densities rapidly became the norm. Pioneering works even showed ${ }^{13,14}$ that many difficult cases for semilocal DFT were improved by using HF densities. Other cases, such as the prototype of stretched $\mathrm{H}_{2}{ }^{+}$, could not be This is a genuine error of the approximate energy functionals and is barely affected by using the exact density.

However, a quantitative and general analysis appeared only recently, ${ }^{7,9}$ showing that many cases of delocalization error could be greatly reduced (if not eliminated) by HF-DFT. This analysis has been used to explain how accurate electron affinities can be extracted from (formally) unconverged DFT calculations, ${ }^{15}$ why barrier heights are usually improved by evaluating functionals on HF densities, ${ }^{13}$ why the apparently very poor-quality potentials of semilocal approximations usually do not matter in the energy, ${ }^{9}$ and many other phenomena. A recent application of DC-DFT to ions and radicals in solution ${ }^{7}$ shows that the potential energy surfaces of choride-water and hydroxide-water complexes are quite inaccurate with standard approximations such as B3LYP but are almost perfect when B3LYP is evaluated on HF densities. Moreover, while different approximations yielded significantly different potential energy surface when performed self-consistently, their agreement is so good in HF-DFT that there is no need to compare with sophisticated quantum-chemical methods like $\operatorname{CCSD}(\mathrm{T}){ }^{7} \mathrm{We}$ note that implementation of HF-DFT is particularly simple for evaluation of energies within any standard quantum chemical code. Simply solve the HF equations as usual, then use the output HF density as the initial guess for a DFT calculation, but stop the code before the density is updated, as described in a Web site (http://tccl.yonsei.ac.kr/mediawiki/index.php/DCDFT).

The present work concerns heteronuclear bond dissociation in semilocal DFT and how to improve it using DC-DFT. (Calculation details are given in Supporting Information.) From very early on, ${ }^{16,17}$ it was recognized that, in many cases, semilocal approximations cause molecules to dissociate into charged fragments. The linearity of the exact functional with particle number ${ }^{18}$ guarantees that all diatomics dissociate into fragments of integer charge. Semilocal approximations are smooth functions of particle number and lack the discontinuity in the derivative of the energy at integers. ${ }^{19}$ This, in turn, means they have an analytic minimum around some nonzero value of charge transfer between the fragments, $q$, in the infinitely stretched limit. (An ensemble generalization ${ }^{20}$ cures this but only in the infinitely stretched limit.) Nowadays, we call this delocalization error. ${ }^{2}$ When $q$ is finite, the energy of the molecule, stretched to $R \rightarrow \infty$, does not match the sum of the energies of the fragments. ${ }^{4}$ In the classic case of $\mathrm{NaCl}$, the stretched molecule is a full $1 \mathrm{eV}$ lower in energy than the fragments in PBE. (In standard practice, this embarrassment is avoided by reporting the binding energy of a molecule as the difference between equilibrium energy and the sum of neutral atomic energies, not the difference between the equilibrium energy and infinitely stretched molecules.)

To explain the correct DC-DFT procedure for heteronuclear diatomics, in Figure 2, we plot several potential energy curves,

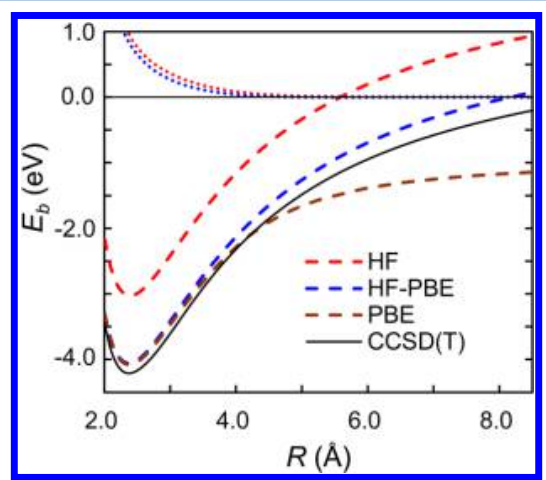

Figure 2. Binding energy curves of $\mathrm{NaCl}$ with various methods and spin multiplicities. The dashed lines are singlet potential energy curves, and dotted lines are triplet potential energy curves. The reference curve $(\operatorname{CCSD}(T))$ is given in a black solid line.

using dashed lines for singlet and dotted lines for triplets. The self-consistent PBE singlet curve (brown) is smooth but goes to the incorrect limit. The HF curves (red) cross at 5.6 $\AA$, where the minimizing HF density switches to the triplet. Thus, PBE, evaluated on the minimizing HF density, produces the cyan curve of Figure 1, but the correct procedure is to switch curves only when $E^{\mathrm{PBE}}\left[\rho_{\mathrm{I}}^{\mathrm{HF}}\right]>E^{\mathrm{PBE}}\left[\rho_{\mathrm{III}}^{\mathrm{HF}}\right]$. Thus, HF-DFT follows the blue dashed curve until $R=8.2 \AA$. Compared with $\operatorname{CCSD}(\mathrm{T})$ (black solid line), it is an almost constant shift prior to $8.2 \AA$.

In Figure 3, we plot Mulliken charges, $Q(R)$, as a function of bond length for $\mathrm{NaCl}$. The value of $Q$ in self-consistent PBE differs only slightly from that of $\mathrm{HF}$ in the region $R=2.0-4.0$ $\AA$, but beyond $4 \AA$, in self-consistent PBE, $Q$ drops slowly toward its asymptotic value of $q=0.4$. The drop is the onset of the density-driven error, as the HOMO-LUMO gap becomes very small as $R$ increases. ${ }^{9}$ This value of $8.2 \AA$ is not much smaller than the value of $9.5 \AA$ for the exact functional, ${ }^{4}$ in which region the charge localized on the ions suddenly drops to 0 . On the contrary, the HF charge keeps increasing toward 1, until it suddenly drops to 0 . This behavior is generic and can be traced back to the behavior of the approximations for fractionally charged fragments. ${ }^{16}$ The exact functional is linear between integer occupations. Semilocal functionals are concave, which causes the minimum of a stretched heteronuclear bond to differ from integer occupations, while $\mathrm{HF}$ is convex, ${ }^{21}$ producing downward cusps at the integer occupations. Usually this is overcomed by using self-interaction-corrected DFT, ${ }^{22}$ 


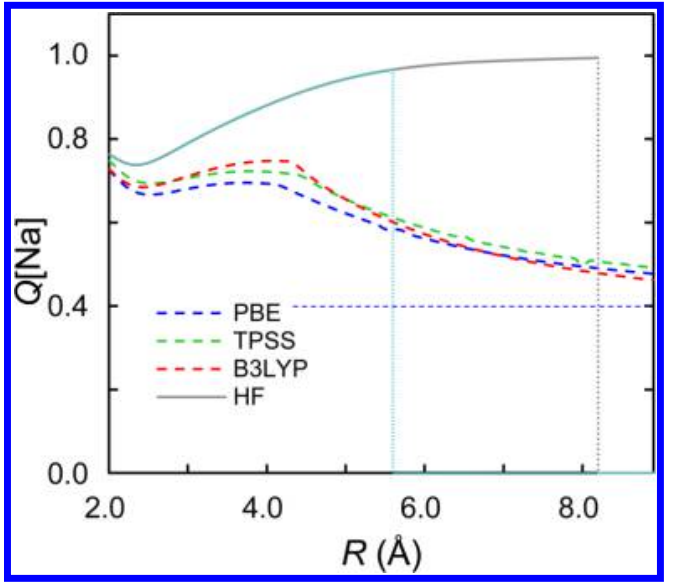

Figure 3. Mulliken charge of $\mathrm{Na}$ in $\mathrm{NaCl}$ from $\mathrm{HF}$ and semilocal DFT calculations. The light-blue line is the HF charge following the previous procedure. ${ }^{9}$

orbital-dependent DFT methods, ${ }^{23}$ hybrid functionals tuned to have correct linear behavior, ${ }^{21,24}$ and so on.

To practice this assumption, we show the results for several different approximations in Figure 4. The two purely semilocal

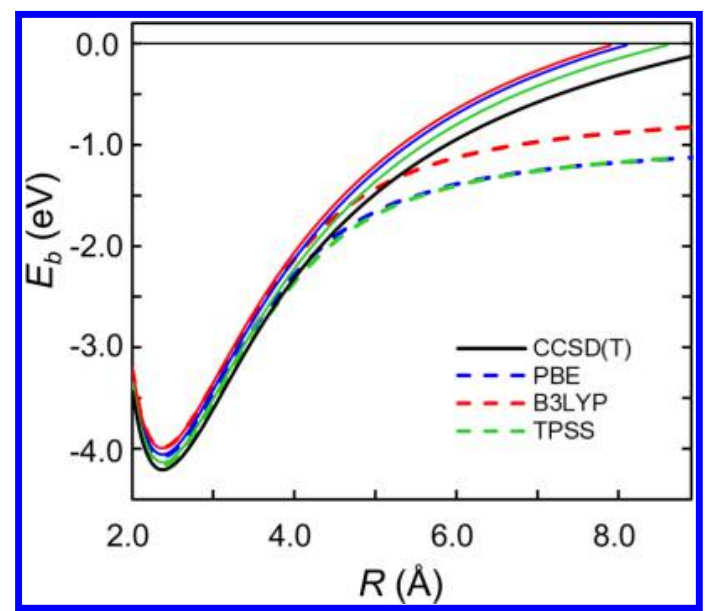

Figure 4. Binding energy curve of $\mathrm{NaCl}$ with various DFT approximations. Dashed lines are self-consistent and solid are HFDFT.

curves, PBE and TPSS, are almost identical at large $R$, both selfconsistently (dashed) and using the new version of HF-DFT (solid). The B3LYP curve, including $20 \%$ exact exchange, does slightly better self-consistently because its density is $\sim 20 \%$ better, but HF-B3LYP is almost identical to HF-PBE, and HFTPSS is slightly better. They are each much closer to the $\operatorname{CCSD}(\mathrm{T})$ curve than their self-consistent analogs and tend toward the right value for large $R$. Moreover, their errors for large $R$ are not much different from their errors at equilibrium.

Next, we apply our method to charged species to test its limitations. For anions, self-consistent DFT with semilocal approximations is notorious for not even binding the last electron, unless it is artificially (but accurately) confined by a limited basis set. ${ }^{25}$ In Figure 5, we plot the binding energy curve of $\mathrm{CH}^{-}$with several local approximations, both selfconsistently and within HF-DFT. The differences between HFDFT and self-consistent DFT are very small at equilibrium, but after $\sim 1.7 \AA$, there is a huge difference. The self-consistent curves are too low and in fact plateau $\sim 1 \mathrm{eV}$ below the

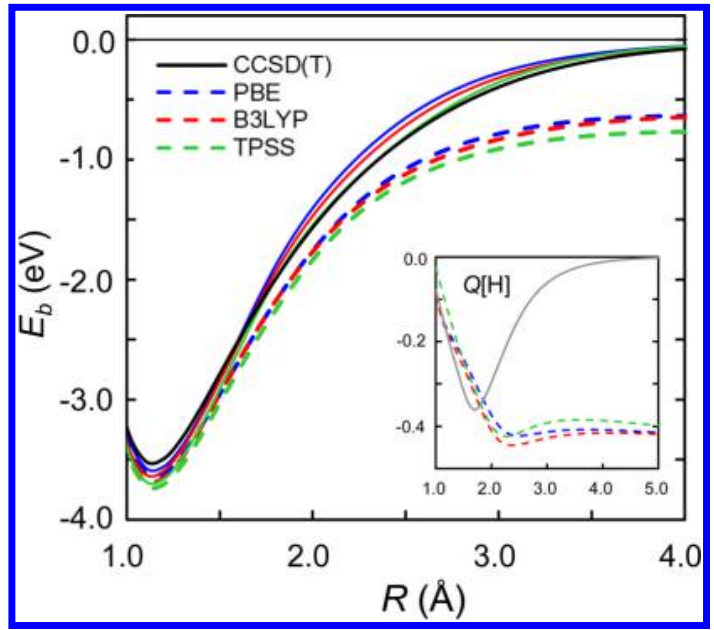

Figure 5. Binding energy curves of $\mathrm{CH}^{-}$in self-consistent and HFDFT. Inset: charge of $\mathrm{H}$ in $\mathrm{CH}^{-}$ion. The gray solid line is the $\mathrm{HF}$ charge.

dissociated fragment energies of $\mathrm{C}^{-}$plus $\mathrm{H}$. On the contrary, the HF-DFT curves are all in close agreement with one another and with the reference $\operatorname{CCSD}(\mathrm{T})$ curve. Again, the Mulliken charges of the various self-consistent calculations show the story. In the inset of Figure 5, we plot these for the $\mathrm{H}$ atom and see that the HF charge loosely agrees with the self-consistent local DFT charge out to $\sim 1.7 \AA$ but differs radically after this. Semilocal DFT allows about $q=|Q[\mathrm{H}]|=0.34$ of an electron to transfer from $\mathrm{C}^{-}$to the $\mathrm{H}$ atom, whereas $\mathrm{HF}$ localizes the extra electron entirely on the $\mathrm{C}$ atom.

We next consider a prototypical example of a molecular cation, $\mathrm{CH}^{+}$. We compare various binding energy curves of $\mathrm{CH}^{+}$in Figure 6 and Figure S1(Supporting Information). It is

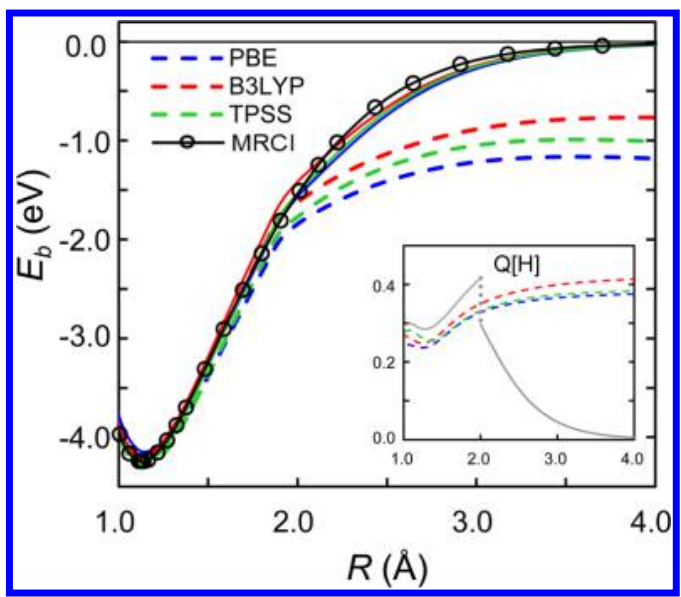

Figure 6. Binding energy curves of $\mathrm{CH}^{+}$in self-consistent and HFDFT. The black circled solid line is MRCI results from ref 22 . Inset: charge of $\mathrm{H}$ in $\mathrm{CH}^{+}$ion. The gray solid line is the $\mathrm{HF}$ charge.

well known that single-reference electronic structure calculations for the dissociation of $\mathrm{CH}^{+}$have difficulties, so we use a recent ground-state multireference configuration interaction (MRCI) result $^{26}$ as reference. Because the atomic MRCI energies of $\mathrm{C}^{+}$are not given, we chose the MRCI energy of $R=$ 30 au $(\sim 15.9 \AA)$ as the zero point. The pattern is very similar to the previous cases. All semilocal curves go to the incorrect dissociation limit $\sim 1.0 \mathrm{eV}$ below 0 , while HF-DFT curves agree well with the MRCI curve. To emphasize this, in Figure 7, we 


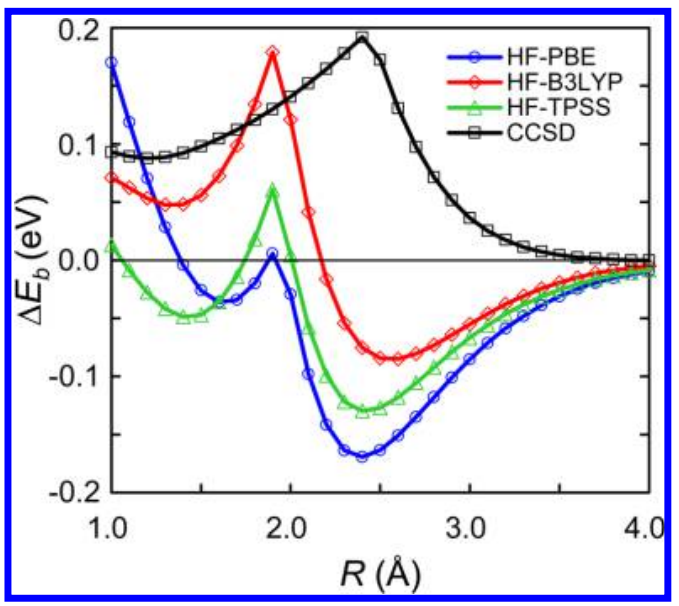

Figure 7. Binding energy error curves of $\mathrm{CH}^{+}$calculated with CCSD and HF-DFT methods. The reference values come from interpolating MRCI results of ref 22 .

plot the errors made by CCSD and the various HF-DFT calculations. (The self-consistent curves have errors off this scale.) Although all curves contain a kink, which is the point where curve crossing between singlet and triplet curve occurs, the magnitude of the error is typically smaller in HF-DFT than in CCSD, being particularly small for HF-TPSS.

So, should we expect all HF-DFT calculations to fix all dissociation problems, yielding accurate binding curves out to very large separations? If not, when do we expect HF-DFT to yield such good curves and when not? How would we know without having to run sophisticated calculations like $\operatorname{CCSD}(\mathrm{T})$, thereby defeating the purpose of these low-cost calculations? We next apply the analysis tools of DC-DFT to answer these questions.

The basic tool is to study the gap of the semilocal DFT calculation. ${ }^{7,9}$ An unusually small self-consistent gap (often 0 or below $0.5 \mathrm{eV}$ ) suggests a possible density-driven error, so a HFDFT calculation should be done to see if energies change significantly. None of the neutral or cationic molecules studied here meet this criterion at equilibrium, implying that semilocal results are normal and barely changed by using HF-DFT. If near-equilibrium properties are the only ones needed, there is no reason to do HF-DFT (and substantial reasons to avoid $\mathrm{it}^{27}$ ). Anions are abnormal at all distances and always improved by HF-DFT. ${ }^{25}$

But in essentially every case, the gap shrinks to zero or nearzero at some finite value of $R$, so that all stretched molecules could be abnormal in semilocal DFT. In fact, the gap of the molecular system in semilocal DFT can be somewhat ambiguous in a stretched molecular system. To find a more useful criterion, we study specifically the stretched limit, that is, where $R$ is sufficiently large that all quantites are close to their limiting values. In this limit, a powerful result for approximate DFT for neutral molecules has long been known. ${ }^{16}$ If the isolated atoms satisfy

$$
\epsilon^{\mathrm{HOMO}}(A)>\epsilon^{\mathrm{LUMO}}(B)
$$

where $A$ and $B$ are either of the atoms, then this solution cannot be a minimum for the stretched molecule, and a selfconsistent calculation will keep transferring charge until these values match one another. We denote that value by $q=I Q(R \rightarrow$ $\infty)$. We have found that this criterion generalizes to molecular ions also. One first chooses the fragments with integer occupations that have lowest energy. Now one of them is an ion. In the case of anions, the LUMO eigenvalues of the semilocal functional are likely to be positive but well-defined with a carefully chosen basis set (as shown in ref 12). In Table S1(Supporting Information), we give a table of atomic neutrals and ions showing the relevant levels.

Using the atomic inputs, we construct Table 1 for the molecules and molecular ions discussed here and other

Table 1. Various Properties of Molecular Species Calculated With PBE $^{a}$

\begin{tabular}{lllllrr} 
& $A$ & $B$ & $q$ & $\epsilon^{\mathrm{HOMO}}[\mathrm{A}]$ & $\epsilon^{\mathrm{LUMO}}[\mathrm{B}]$ & \multicolumn{1}{c}{$\Delta \epsilon_{\mathrm{P}}$} \\
$\mathrm{NH}$ & $\mathrm{H}$ & $\mathrm{N}$ & 0.00 & -7.59 & -4.13 & -3.46 \\
$\mathrm{NO}$ & $\mathrm{N}$ & $\mathrm{O}$ & 0.00 & -8.30 & -6.49 & -1.82 \\
$\mathrm{HS}$ & $\mathrm{H}$ & $\mathrm{S}$ & 0.00 & -7.59 & -5.64 & -1.96 \\
$\mathrm{CH}$ & $\mathrm{H}$ & $\mathrm{C}$ & 0.00 & -7.59 & -5.58 & -2.01 \\
$\mathrm{NaH}$ & $\mathrm{H}$ & $\mathrm{Na}$ & 0.00 & -7.59 & -1.17 & -6.42 \\
$\mathrm{HF}$ & $\mathrm{H}$ & $\mathrm{F}$ & 0.09 & -7.59 & -9.30 & 1.70 \\
$\mathrm{PCl}$ & $\mathrm{P}$ & $\mathrm{Cl}$ & 0.14 & -6.30 & -7.71 & 1.42 \\
$\mathrm{NaCl}$ & $\mathrm{Na}$ & $\mathrm{Cl}$ & 0.32 & $-3.04^{b}$ & -7.73 & 4.69 \\
$\mathrm{AlO}$ & $\mathrm{Al}$ & $\mathrm{O}$ & 0.33 & -3.10 & -6.44 & 3.35 \\
$\mathrm{NaO}$ & $\mathrm{Na}$ & $\mathrm{O}$ & 0.38 & -3.04 & -6.49 & 3.45 \\
$\mathrm{CaF}$ & $\mathrm{Ca}$ & $\mathrm{F}$ & 0.51 & $-3.75^{b}$ & -9.25 & 5.50 \\
$\mathrm{NaO}$ & $\mathrm{O}$ & $\mathrm{Na}$ & 0.00 & -7.60 & -7.23 & -0.38 \\
$\mathrm{SiN}$ & $\mathrm{N}$ & $\mathrm{Si}$ & 0.06 & -8.30 & -11.81 & 3.50 \\
$\mathrm{BH}^{+}$ & $\mathrm{H}$ & $\mathrm{B}^{+}$ & 0.19 & -7.59 & -13.36 & 5.77 \\
$\mathrm{CH}^{+}$ & $\mathrm{H}$ & $\mathrm{C}^{+}$ & 0.37 & -7.59 & -17.15 & 9.55 \\
$\mathrm{NO}^{+}$ & $\mathrm{N}$ & $\mathrm{O}^{+}$ & 0.47 & -8.30 & -20.61 & 12.30 \\
$\mathrm{HF}^{-}$ & $\mathrm{F}^{-}$ & $\mathrm{H}$ & 0.28 & 1.47 & 0.27 & 1.20 \\
$\mathrm{NO}^{-}$ & $\mathrm{O}^{-}$ & $\mathrm{N}$ & 0.33 & 2.38 & -4.13 & 6.52 \\
$\mathrm{CH}^{-}$ & $\mathrm{C}^{-}$ & $\mathrm{H}$ & 0.41 & 1.81 & 0.27 & 1.54 \\
$\mathrm{NH}^{-}$ & $\mathrm{N}^{-}$ & $\mathrm{H}$ & 0.56 & 2.93 & 0.27 & 2.66
\end{tabular}

${ }^{a}$ Amount of charge transfer in the dissociation limit, $q$, is calculated at $R=5.0 \AA$ and with the def2-QZVP basis. ${ }^{28} \mathrm{~A}$ is for electropositive atomic species, while $\mathrm{B}$ is for electronegative ones. If not specified, $\epsilon$ is calculated with the aug-cc-pV5Z basis set. ${ }^{29}{ }^{b}$ Calculated with the ccpV5Z basis set. ${ }^{30}$

examples. The HOMO-LUMO criterion eq 2 applies in all cases for large $R$ : If it is satisfied, $q$ is finite, and semilocal DFT tends to an incorrect energy at large $R$. By incorrect, we mean different from the sum of the energies of the isolated fragments. The stretched molecule is abnormal and is usually improved by a HF-DFT calculation, as shown in our figures.

An important point is that this analysis applies only in the stretched limit. Even if a molecule is normal in this limit, that is, has $q=0$, it still has a small or vanishing gap for finite $R$ and must be checked for abnormality at finite $R$. In such cases, HFDFT might still yield an accurate binding energy curve out to larger $R$ than self-consistent DFT, but we do not have a simple condition for predicting when this will be the case.

Figure 8 shows a typical case of a normal calculation. Semilocal functionals all go correctly to the zero dissociation limit, and HF-DFT worsens the result for all $R$. The charges do not vary much and vanish at large $R$ regardless of the functional you use. There are also some cases where the abnormality depends on the particular semilocal DFT calculation. Consider HF in Figure 9. From our table, PBE transfers a small charge at large $R$, making it abnormal, and the PBE binding energy curve is improved by HF-PBE. This also holds for TPSS, where HFTPSS slightly improves the results, but this is not true for B3LYP, so HF-B3LYP does not improve its result. 


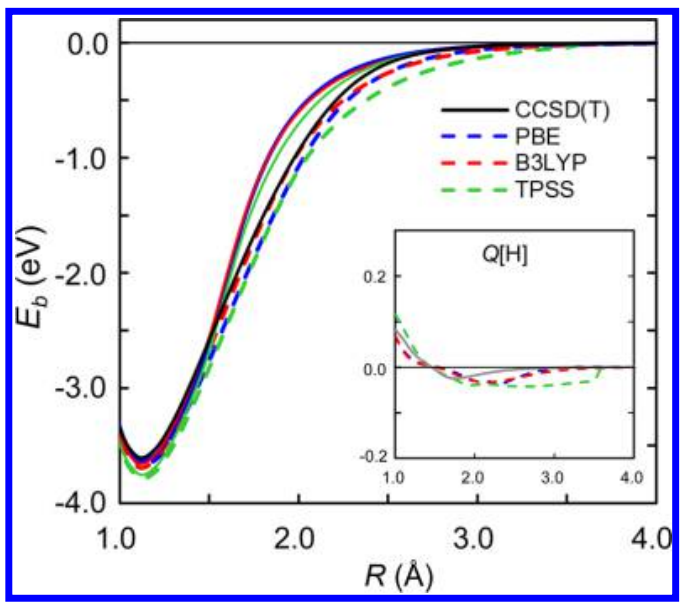

Figure 8. Binding energy curves of $\mathrm{CH}$ in self-consistent and HFDFT. Inset: charge of $\mathrm{H}$ in $\mathrm{CH}$ molecule. The gray solid line is the HF charge.

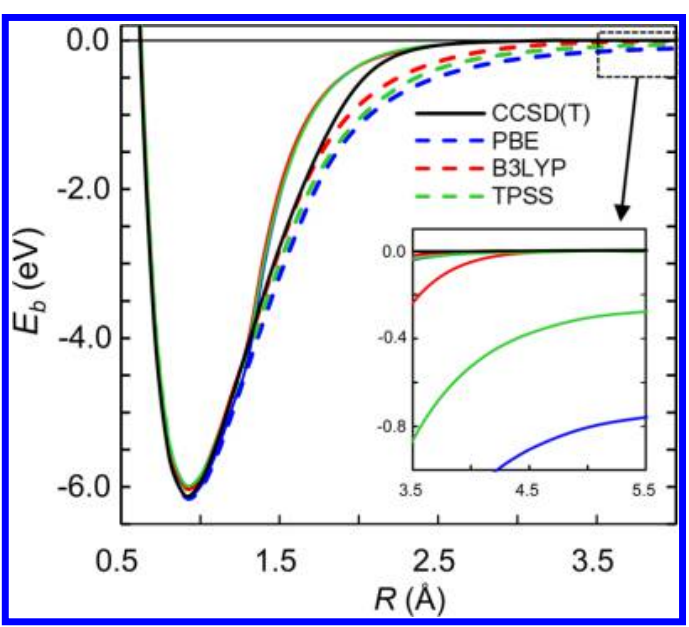

Figure 9. Binding energy curves of $\mathrm{HF}$ evaluated with various methods. Inset: blowup.

We have commented only on heteronuclear diatomics, even though the classic cases of breakdown of DFT due to delocalization error are homogeneous (stretched $\mathrm{H}_{2}{ }^{+}$and $\mathrm{H}_{2}$ ). This is because we study only abnormal calculations and how to fix them. The classic examples do not have large density-driven errors.

To conclude, considerable evidence shows that, in many cases, semilocal DFT errors in heteronuclear binding energy curves are density-driven and can be reduced by using HFDFT. We have significantly extended the range of DC-DFT beyond previous suggestions and have given a clear criterion for when DC-DFT will be better than self-consistent DFT. In the infinitely stretched limit, DC-DFT always yields the correct dissociation, which is the sum of the fragment energies (within DC-DFT). If a self-consistent DFT density incorrectly transfers charge, the HF density will be better. While this is not an ideal procedure in terms of model chemistries or a black box that can be applied unambiguously to every problem, DC-DFT provides a pragmatic approach for those who need dissociation curves at larger distances while retaining a DFT-level of computational cost as well as food for thought for more fundamental theory development.

\section{ASSOCIATED CONTENT}

\section{S Supporting Information}

The Supporting Information is available free of charge on the ACS Publications website at DOI: 10.1021/acs.jpclett.5b01724.

Computational details and frontier orbital energies of atomic species. (PDF)

\section{AUTHOR INFORMATION}

\section{Corresponding Author}

*E-mail: esim@yonsei.ac.kr.

Notes

The authors declare no competing financial interest.

\section{ACKNOWLEDGMENTS}

This work was supported by grants from the National Research Foundation (2014R1A1A3049671 and 2012R1A1A2004782) by the Korean government and by the Yonsei Future-Leading Research Initiative (2014-22-0169). K.B. acknowledges NSF CHE 1464795.

\section{REFERENCES}

(1) Pribram-Jones, A.; Gross, D. A.; Burke, K. DFT: A Theory Full of Holes? Annu. Rev. Phvs. Chem. 2015, 66, 283-304.

(2) Cohen, A. J.; Mori-Sánchez, P.; Yang, W. Insights into Current Limitations of Density Functional Theory. Science 2008, 321, 792794.

(3) Li, C.; Zheng, X.; Cohen, A. J.; Mori-Sánchez, P.; Yang, W. Local Scaling Correction for Reducing Delocalization Error in Density Functional Approximations. Phvs. Rev. Lett. 2015, 114, 053001.

(4) Ruzsinszky, A.; Perdew, J. P.; Csonka, G. I.; Vydrov, O. A.; Scuseria, G. E. Spurious Fractional Charge on Dissociated Atoms: Pervasive and Resilient Self-Interaction Error of Common Density Functionals. I. Chem. Phvs. 2006, 125, 194112.

(5) Perdew, J. P.; Burke, K.; Ernzerhof, M. Generalized Gradient Approximation Made Simple. Phys. Rev. Lett. 1996, 77, 3865-3868.

(6) Grimme, S. Semiempirical GGA-type density functional constructed with a long-range dispersion correction. J. Comput. Chem. 2006, 27, 1787-1799.

(7) Kim, M.-C.; Sim, E.; Burke, K. Ions in Solution: Density Corrected Density Functional Theory (DC-DFT). J. Chem. Phys. 2014, 140, 18A528.

(8) Kohn, W.; Sham, L. J. Self-Consistent Equations Including Exchange and Correlation Effects. Phys. Rev. 1965, 140, A1133A1138.

(9) Kim, M.-C.; Sim, E.; Burke, K. Understanding and Reducing Errors in Density Functional Calculations. Phys. Rev. Lett. 2013, 111, 073003.

(10) Shore, H. B.; Rose, J. H.; Zaremba, E. Failure of the Local Exchange Approximation in the Evaluation of the $\mathrm{H}^{-}$Ground State. Phys. Rev. B 1977, 15, 2858.

(11) Jarecki, A. A.; Davidson, E. R. Density Functional Theory Calculations for F. Chem. Phvs. Lett. 1999, 300, 44-52.

(12) Gill, P. M. W.; Johnson, B. G.; Pople, J. A.; Frisch, M. J. An Investigation of the Performance of a Hybrid of Hartree-Fock and Density Functional Theory. Int. J. Quantum Chem. 1992, 44, 319-331.

(13) Janesko, B. G.; Scuseria, G. E. Hartree-Fock Orbitals Significantly Improve the Reaction Barrier Heights Predicted by Semilocal Density Functionals. J. Chem. Phys. 2008, 128, 244112.

(14) Verma, P.; Perera, A.; Bartlett, R. J. Increasing the Applicability of DFT I: Non-variational Correlation Corrections from Hartree-Fock DFT for Predicting Transition States. Chem. Phys. Lett. 2012, 524, 1015 .

(15) Lee, D.; Furche, F.; Burke, K. Accuracy of Electron Affinities of Atoms in Approximate Density Functional Theory. J. Phys. Chem. Lett. 2010, 1, 2124-2129. 
(16) Perdew, J. In Density Functional Methods in Physics; Dreizler, R., da Providencia, J., Eds.; Plenum: New York, 1985; p 265.

(17) Bally, T.; Sastry, G. N. Incorrect Dissociation Behavior of Radical Ions in Density Functional Calculations. J. Phys. Chem. A 1997, 101, 7923-7925.

(18) Perdew, J. P.; Parr, R. G.; Levy, M.; Balduz, J. L. DensityFunctional Theory for Fractional Particle Number: Derivative Discontinuities of the Energy. Phvs. Rev. Lett. 1982, 49, 1691-1694.

(19) Dreizler, R. M.; Gross, E. K. U. Density Functional Theory: An Approach to the Quantum Many-Body Problem; Springer-Verlag: Berlin, 1990.

(20) Kraisler, E.; Kronik, L. Elimination of the Asymptotic Fractional Dissociation Problem in Kohn-Sham Density Functional Theory Using the Ensemble-Generalization Approach. Phys. Rev. A: At., Mol., Opt. Phys. 2015, 91, 032504.

(21) Cohen, A. J.; Mori-Sánchez, P.; Yang, W. Development of Exchange-Correlation Functionals with Minimal Many-Electron SelfInteraction Error. J. Chem. Phys. 2007, 126, 191109.

(22) Perdew, J. P.; Zunger, A. Self-Interaction Correction to DensityFunctional Approximations for Many-Electron Systems. Phys. Rev. B: Condens. Matter Mater. Phvs. 1981, 23, 5048-5079.

(23) Erhart, P.; Klein, A.; Åberg, D.; Sadigh, B. Efficacy of the DFT + $U$ Formalism for Modeling Hole Polarons in Perovskite Oxides. Phys. Rev. B: Condens. Matter Mater. Phvs. 2014, 90, 035204.

(24) Atalla, V.; Yoon, M.; Caruso, F.; Rinke, P.; Scheffler, M. Hybrid Density Functional Theory Meets Quasiparticle Calculations: A Consistent Electronic Structure Approach. Phys. Rev. B: Condens. Matter Mater. Phvs. 2013, 88, 165122.

(25) Kim, M.-C.; Sim, E.; Burke, K. Communication: Avoiding Unbound Anions in Density Functional Calculations. J. Chem. Phys. 2011, 134, 171103.

(26) Biglari, Z.; Shayesteh, A.; Maghari, A. Ab Initio Potential Energy Curves and Transition Dipole Moments for the Low-Lying States of $\mathrm{CH}^{+}$. Comput. Theor. Chem. 2014, 1047, 22-29.

(27) Vassilev, P.; Louwerse, M. J.; Baerends, E. J. Hydroxyl Radical and Hydroxide Ion in Liquid Water: A Comparative Electron Density Functional Theory Study. J. Phys. Chem. B 2005, 109, 23605-23610.

(28) Weigend, F.; Ahlrichs, R. Balanced Basis Sets of Split Valence, Triple Zeta Valence and Quadruple Zeta Valence Quality for H to Rn: Design and Assessment of Accuracy. Phys. Chem. Chem. Phys. 2005, 7, 3297-3305.

(29) Woon, D. E.; Dunning, T. H., Jr. Gaussian Basis Sets for Use in Correlated Molecular Calculations. III. The Atoms Aluminum through Argon. I. Chem. Phys. 1993, 98, 1358-1371.

(30) Prascher, B. P.; Woon, D. E.; Peterson, K. A.; Dunning, T. H., Jr.; Wilson, A. K. Gaussian Basis Sets for Use in Correlated Molecular Calculations. VII. Valence, Core-Valence, and Scalar Relativistic Basis Sets for $\mathrm{Li}, \mathrm{Be}, \mathrm{Na}$, and $\mathrm{Mg}$. Theor. Chem. Acc. 2011, 128, 69-82. 